\title{
Search for gluonic excitations in light unconventional mesons
}

\section{Paul Eugenio*}

Florida State University, Department of Physics, Tallahassee, Florida 32306 USA

E-mail: eugenio@fsu.edu

\begin{abstract}
Studies of meson spectra via strong decays provide insight regarding QCD at the confinement scale. These studies have led to phenomenological models for QCD such as the constituent quark model. However, QCD allows for a much richer spectrum of meson states which include extra states such as exotics, hybrids, multi-quarks, and glueballs. First discussion of the status of exotic meson searches is given followed by a discussion of plans at Jefferson Lab to double the energy of the machine to $12 \mathrm{GeV}$, which will allow us to access photoproduction of mesons in search for gluonic excited states.
\end{abstract}

XXIV International Symposium on Lattice Field Theory

July 23-28 2006

Tucson Arizona, US

${ }^{*}$ Speaker. 


\section{Introduction}

Discoveries of new phenomena in nuclear and particle physics have provided insight into the fundamental constituents of matter. In the past few decades we have seen a new picture emerge in which quarks form the building blocks of nearly all matter. Yet the gluon, which carries the force which binds quarks, can interact with other gluons to form a bound state, or interact as a fundamental constituent of matter along with the quarks. Thus new forms of gluonic or hybrid matter should exist.

The search for hybrids in recent years has resulted in considerable excitement. Theoretical predictions from both gluonic flux-tube models and recent lattice gauge theory results predict the lightest hybrid at a mass of $1.9 \mathrm{GeV}$ for the exotic $J^{P C}=1^{-+} q \bar{q} g$-hybrid(see references [1] and [2]). Exotic meson states are those with quantum numbers not accessible to conventional $q \bar{q}$ bound states. Recent experimental results find two very promising $1^{-+}$exotic candidates. The $\pi_{1}(1400)$ seen decaying to $\eta \pi^{-}$at Brookhaven has a mass somewhat too low for the theory prediction for a gluonic hybrid [3]. A higher mass observed state, the $\pi_{1}(1600)$ is tantalizing as a gluonic hybrid, but its decay to $\rho \pi$ was unexpected [4]. Even though the existence of both states appears very clear, these states have had a history of controversy, particular those produced via pion beams [5].

It has been pointed out by Close and Page [6] that in the case of photoproduction, where the photon can be effectively replaced by a $\rho$ interacting with an exchange $\pi, \rho$, or $\omega$, the production strength for producing gluonic hybrids could be considerable. Furthermore, Szczepaniak and Swat [7] concluded that in the case of photoproduction, the $\pi_{1}$ exotic and the well known $a_{2}$ should be produced on an equal footing, whereas in pion production the exotic is suppressed by a factor of 10 .

As supported by recent lattice gauge calculations using excited adiabatic potentials, it is a good approximation to decouple the quark degrees of freedom from the gluonic degrees of freedom. This is based on the idea that quarks in the systems react much slower than the gluonic fields responsible for strong confinement. As in ordinary $q \bar{q}$ mesons where the addition of one unit of orbital angular momentum costs about $1 \mathrm{GeV}$, calculations show that gluonic excitations are also of the order of 1 $\mathrm{GeV}$ in energy, and that there is a kind of orthogonality decoupling the quarks and flux-tube (gluon) degrees of freedom. In lattice QCD calculations and in flux-tube models, excited flux-tubes can have

$$
\text { flux-tube } J^{P C}=1^{+-} \text {or } 1^{-+}[8] .
$$

Therefore with pseudoscalar probes, such as pion beams, coupling the quark degrees of freedom to those of an excited flux-tube results in

$$
{ }_{\text {quarks }} J^{P C} \otimes_{\text {flux-tube }} J^{P C}=1^{--}, 1^{++} .
$$

On the other hand, for vector probes, such as the photon viewed as a vector meson, coupling the quark degrees of freedom with that of an excited flux-tube results in

$$
{ }_{\text {quarks }} J^{P C} \otimes_{\text {flux-tube }} J^{P C}=0^{-+}, 1^{-+}, 2^{-+}, 0^{+-}, 1^{+-}, 2^{+-} .
$$

It is interesting to note that the vector probe has access to manifestly exotic quantum numbers (exotic $J^{P C}=0^{+-}, 1^{-+}, 2^{+-}$). Since there exists a wealth of data with pseudoscalar hadronic probes 


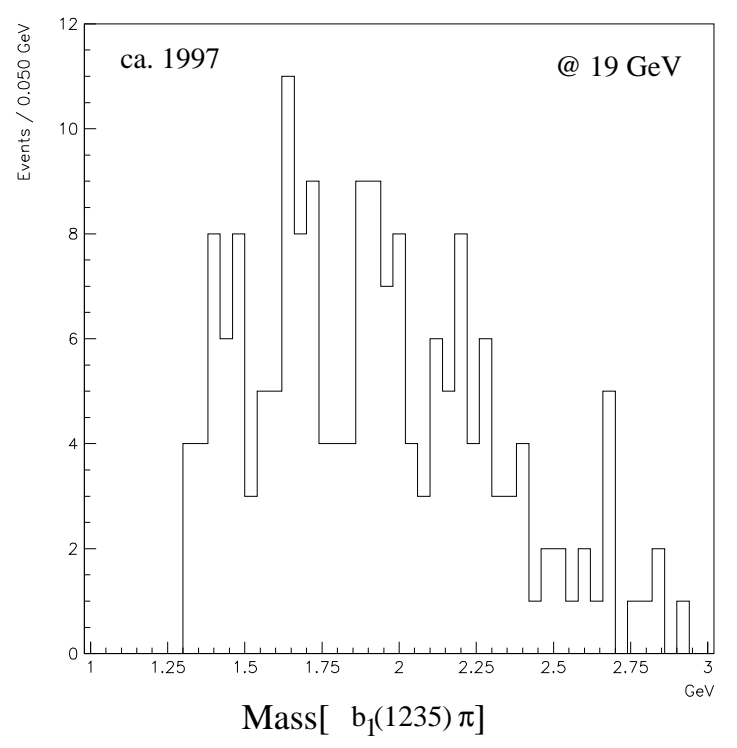

Figure 1: The $b_{1}(1235) \pi$ mass spectrum from $\gamma p \rightarrow p \pi^{+} \pi^{+} \pi^{-} \pi^{-} \pi^{0}$ at $16.5-21.0 \mathrm{GeV}$.

and very little data with vector probes like the photon, this may explain the lack of observations of gluonic hybrids.

Flux-tube model calculations of gluonic hybrid decays prefer decay channels to $(\mathrm{L}=0)+(\mathrm{L}=1)$ meson pairs. For example, according to these calculations the lowest lying exotic state $\left(J^{P C}=1^{-+}\right)$ should have typical partial widths [10]

$$
b_{1} \pi: f_{1} \pi: \rho \pi=170 \mathrm{MeV}: 60 \mathrm{MeV}: 10 \mathrm{MeV} .
$$

Figure 1 shows the $b_{1} \pi$ invariant mass for a recent analysis of SLAC photoproduction data [11]. The sparse data clearly demonstrates the need for better photoproduction experiments.

Studies are underway to pursue gluonic matter at nuclear physics labs around the world. At JLab there are plans to upgrade the accelerator by doubling its maximum beam energy. A linchpin for the Lab's upgrade plans is the addition of a new experimental program called GlueX. The goal of GlueX is to unambiguously discover and study gluonic matter. Although GlueX will be the definitive experiment for studying gluonic matter, it will be several years until this experimental program begins to acquire data. In the meantime, there is timely and complementary experimental program, HyCLAS, in meson spectroscopy to search for new and unusual mesons via photoproduction using the CLAS detection facility at Jefferson Lab.

\section{Partial Wave Analysis}

\section{Intensity Distributions}

The differential cross-section for a photoproduction reaction determines the intensity distribution, $\mathrm{I}(\tau)$, and therefore the number of events as a function of total mass and $t$. This intensity 


\title{
Isobar Decay to 3 Particles
}

\author{
$X \rightarrow y p_{1} \rightarrow p_{1} p_{2} p_{3}$
}

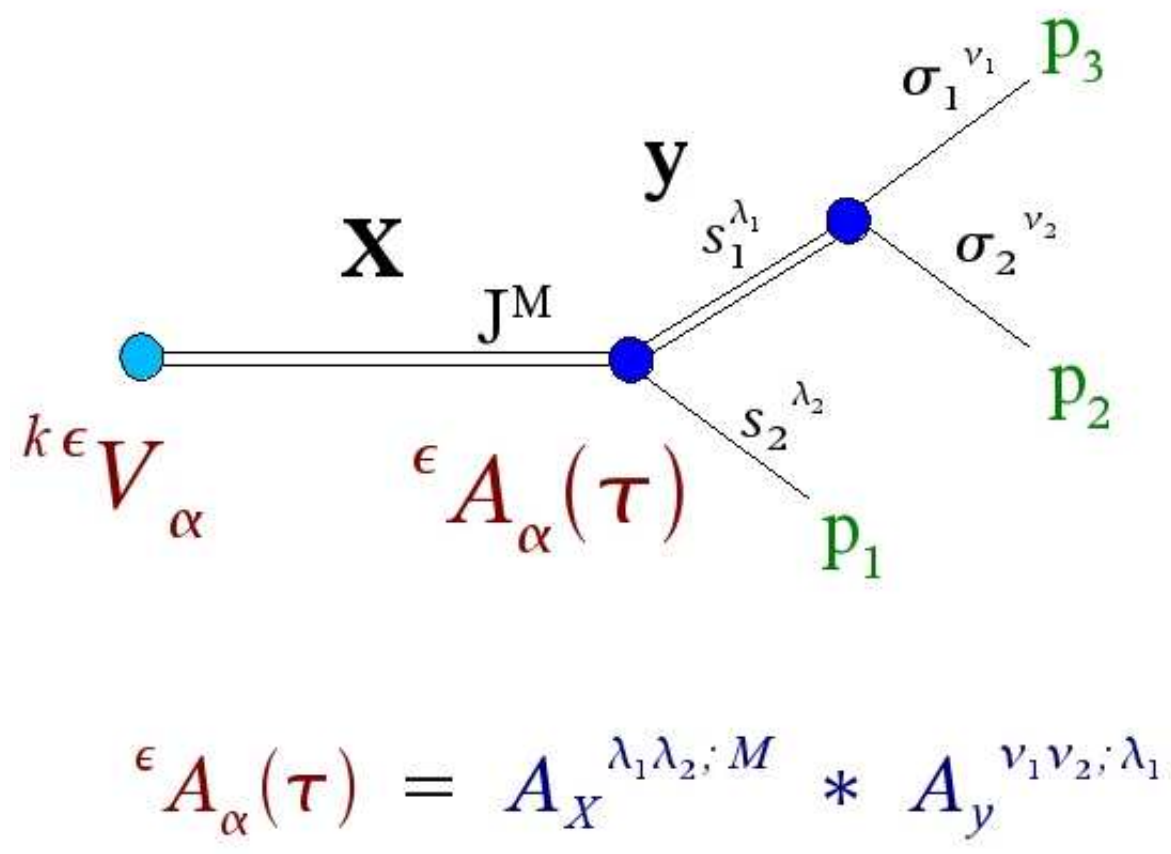

\section{Decay Amplitude}

Figure 2: Isobar decay to three final state particles. The calculation of the decay amplitudes ${ }^{\varepsilon} A_{\alpha}(\tau)$ is based primarily on conservation of angular momentum and parity, with limited phenomenological input from the Isobar Model. The production amplitudes ${ }^{k \varepsilon} V_{\alpha}$ are the complex production strengths. The production amplitudes are the unknown complex parameters which are varied during a partial wave analysis fit to best describe the observed intensity distribution.

distribution is one of the major observables that a partial wave analysis(PWA) attempts to match. It is necessary to perform a PWA to clearly identify the $J^{P C}$ quantum numbers of a meson resonance. A partial wave analysis fits the measured observables such as angular and kinematic distributions to determine the production strength of each partial wave. For a photon beam, the intensity distribution can be written as[13]

$$
I(\tau)=\sum_{k \varepsilon \varepsilon^{\prime}} \varepsilon \varepsilon^{\prime} \rho_{\varepsilon \varepsilon^{\prime}}(\tau) \sum_{\alpha \alpha^{\prime}}{ }^{k \varepsilon^{\prime}} V_{\alpha^{\prime}}^{*}{ }^{\varepsilon^{\prime}} A_{\alpha^{\prime}}^{*}(\tau){ }^{k \varepsilon} V_{\alpha}{ }^{\varepsilon} A_{\alpha}^{*}(\tau)
$$

The intensity distribution is characterized by a set of interfering (denoted by $\alpha$ and $\varepsilon$ ) and noninterfering (denoted by $k$ ) orthonormal amplitudes. The variable $\tau$ represents collectively all the variables necessary to have a complete description of the final state. Often $\tau$ includes the produced 
and intermediate meson masses, decay angles, and four-momentum transfer $t$. The superscript $k$ represents the initial and final spin orientations in the production process. The above amplitudes are expressed in the reflectivity basis (denoted by $\varepsilon$ ), which takes into account parity conservation in the production process by a transformation of helicity states to eigenstates of the reflection operator in the production plane. The photon spin-density matrix in the reflectivity basis is

$$
\rho_{\varepsilon \varepsilon^{\prime}}(\tau)=\left(\begin{array}{cc}
\frac{1}{2}\left(1-P\left[\cos ^{2}(\delta)-\sin ^{2}(\delta)\right]\right) & P \cos (\delta) \sin (\delta) \\
P \cos (\boldsymbol{\delta}) \sin (\boldsymbol{\delta}) & \frac{1}{2}\left(1+P\left[\cos ^{2}(\boldsymbol{\delta})-\sin ^{2}(\boldsymbol{\delta})\right]\right)
\end{array}\right)
$$

Note that states with different reflectivity, $\varepsilon$, only interfere through the off-diagonal elements of the photon spin-density matrix. For unpolarized photons $(P=0)$, states with different reflectivity do not interfere at all.

Following the prescription of the isobar model, the total amplitude is factorized into a production amplitude ${ }^{k \varepsilon} V_{\alpha}$, and a decay amplitude ${ }^{\varepsilon} A_{\alpha}(\tau)$. The calculation of the decay amplitudes ${ }^{\varepsilon} A_{\alpha}(\tau)$ is based primarily on conservation of angular momentum and parity, with limited phenomenological input from the Isobar Model. In the reflectivity basis, the decay amplitudes are given by [12]

$$
{ }^{\varepsilon} A_{\alpha}(\tau)=\Theta(M)\left\{A_{\alpha}^{J M}(\tau)-\varepsilon P_{X}(-1)^{P_{X}-M} A_{\alpha}^{J-M}(\tau)\right\}
$$

where $\Theta(M)=\frac{1}{\sqrt{2}}, \frac{1}{2}, 0$ for $M>0, M=0$, and $M<0$ respectively. $P_{X}$ is the parity of the meson system, and $J, M$ are the usual total meson angular momentum and its projection. The total helicity decay amplitude is a product of decay amplitudes for each sequential decay in the decay chain ( $\left.A_{\alpha}^{J M}(\tau)=A_{X}^{\lambda_{1} \lambda_{2} ; M} * A_{y}^{v_{1} \nu_{2} ; \lambda_{1}} * \ldots\right)$ where $\lambda=\lambda_{1}-\lambda_{2}$ is the total two-body CM helicity(See Figure 2. At each vertex the decay amplitude is given in terms of a Wigner D-function, Clebsch-Gordan Coefficients, and a mass dependent factor $K$ which is often of a Breit-Wigner or K-Matrix form

$$
A_{X}^{\lambda_{1} \lambda_{2} ; M}=D_{\lambda M}^{J}(\theta, \phi) \frac{\sqrt{L(L+1)}}{\sqrt{J(J+1)}}(L 0 ; S \lambda \mid J \lambda)\left(S_{1} \lambda_{1} ; S_{2}-\lambda_{2} ; \mid S \lambda\right) K
$$

The production amplitudes ${ }^{k \varepsilon} V_{\alpha}$ are the complex production strengths. These are the unknown complex parameters which are varied during a partial wave analysis fit to best describe the observed intensity distribution.

\section{Extended Maximum Likelihood Fit}

An extended maximum likelihood fit is used to determine the production amplitudes that best describe the data. The program that performs the fit uses the CERNLIB routine Minuit to maximize the log of the likelihood[14].

The likelihood function for finding $N_{o}$ observed events in a given bin is

$$
\mathscr{L}=e^{-\bar{N}_{o}\left({ }^{\varepsilon} V_{k \alpha}\right)} \prod_{i}^{N_{o}} F\left({ }^{\varepsilon} V_{k \alpha} ; \tau_{i}\right)
$$

where

$$
F\left({ }^{\varepsilon} V_{k \alpha} ; \tau_{i}\right)=I\left(\tau_{i}\right)=\sum_{k, \varepsilon, \alpha, \alpha^{\prime}}{ }^{\varepsilon} V_{k \alpha}{ }^{\varepsilon} V_{k \alpha^{\prime}}^{*} A_{\alpha}\left(\tau_{i}\right)^{\varepsilon} A_{\alpha^{\prime}}^{*}\left(\tau_{i}\right)
$$


is the intensity distribution and

$$
\overline{N_{o}}\left({ }^{\varepsilon} V_{k \alpha}\right)=\int I(\tau) \eta(\tau) p q d \tau=\sum_{k, \varepsilon, \alpha, \alpha^{\prime}}{ }^{\varepsilon} V_{k \alpha}{ }^{\varepsilon} V_{k \alpha^{\prime}}^{*} \int{ }^{\varepsilon} A_{\alpha}(\tau)^{\varepsilon} A_{\alpha^{\prime}}^{*}(\tau) \eta(\tau) p q d \tau
$$

is the average number of events one would observe if the same experiment were repeated many times[15]. The experimental acceptance is represented by $\eta(\tau)$, and $p q d \tau$ represent the Lorentzinvariant phase-space element.

The integrals over the decay amplitudes are often called the accepted normalization integrals[16],

$$
{ }^{\varepsilon} \Phi_{\alpha \alpha^{\prime}}^{\eta}=\int{ }^{\varepsilon} A_{\alpha}(\tau)^{\varepsilon} A_{\alpha^{\prime}}^{*}(\tau) \eta(\tau) p q d \tau
$$

Since the acceptance $\eta(\tau)$ is not known analytically, the accepted normalization integrals are evaluated using Monte Carlo events where the effects of the experiment are modeled to account for the experimental acceptance

$$
{ }^{\varepsilon} \Phi_{\alpha \alpha^{\prime}}^{\eta}=\frac{1}{N_{r}} \sum_{i}^{N_{\eta}}{ }^{\varepsilon} A_{\alpha}\left(\tau_{i}\right)^{\varepsilon} A_{\alpha^{\prime}}^{*}\left(\tau_{i}\right) .
$$

The sum is over the accepted Monte Carlo data, where $N_{r}$ is the number of raw Monte Carlo events generated to end up with a set of $N_{\eta}$ accepted events. The phase-space factor is taken into account by generating the Monte Carlo events according to a phase-space distribution.

The extended log-likelihood function which is maximized now becomes

$$
\begin{gathered}
\ln \mathscr{L}=\sum_{i}^{N_{o}} \ln \left(F\left({ }^{\varepsilon} V_{k \alpha} ; \tau_{i}\right)\right)-\overline{N_{o}}\left({ }^{\varepsilon} V_{k \alpha}\right) \\
\ln \mathscr{L}=\sum_{i}^{N_{o}} \ln \left\{\sum_{k, \varepsilon, \alpha, \alpha^{\prime}}{ }^{\varepsilon} V_{k \alpha}{ }^{\varepsilon} V_{k \alpha^{\prime}}^{*}{ }^{\varepsilon} A_{\alpha}(\tau)^{\varepsilon} A_{\alpha^{\prime}}^{*}(\tau)\right\}-\sum_{k, \varepsilon, \alpha, \alpha^{\prime}}{ }^{\varepsilon} V_{k \alpha}{ }^{\varepsilon} V_{k \alpha^{\prime}}^{*}{ }^{\varepsilon} \Phi_{\alpha \alpha^{\prime}}^{\eta} .
\end{gathered}
$$

The average number of events corrected for acceptance $\left(N_{c}\right)$ can be calculated using the set of $N_{r}$ raw Monte Carlo events along with the fitted results for the production amplitudes by the following relation

$$
\overline{N_{c}}\left({ }^{\varepsilon} V_{k \alpha}\right)=\int I(\tau) p q d \tau=\sum_{k, \varepsilon, \alpha, \alpha^{\prime}}{ }^{\varepsilon} V_{k \alpha}{ }^{\varepsilon} V_{k \alpha^{\prime}}^{*} \Phi_{\alpha \alpha^{\prime}}
$$

where

$$
{ }^{\varepsilon} \Phi_{\alpha \alpha^{\prime}}=\frac{1}{N_{r}} \sum_{i}^{N_{r}} A_{\alpha}(\tau)^{\varepsilon} A_{\alpha^{\prime}}^{*}(\tau)
$$

are the raw normalization integrals. They are calculated just like the accepted normalization integrals except that the Monte Carlo events are not subjected to a simulation of the experimental apparatus.

In practice, the production amplitudes are renormalized via

$$
V \rightarrow \sqrt{N_{o} \frac{N_{r}}{N_{\eta}}} V
$$


Neglecting the constant log term, the log-likelihood function becomes

$$
\ln \mathscr{L}=\sum_{i}^{N_{o}} \ln \left(\sum_{k, \varepsilon, \alpha, \alpha^{\prime}}{ }^{\varepsilon} V_{k \alpha}{ }^{\varepsilon} V_{k \alpha^{\prime}}^{*} A_{\alpha}(\tau)^{\varepsilon} A_{\alpha^{\prime}}^{*}(\tau)\right)-N_{o} \frac{N_{r}}{N_{\eta}} \sum_{k, \varepsilon, \alpha, \alpha^{\prime}}{ }^{\varepsilon} V_{k \alpha}{ }^{\varepsilon} V_{k \alpha^{\prime}}^{*} \Phi_{\alpha \alpha^{\prime}}^{\eta} .
$$

It is advantageous to renormalize because, to first order, the renormalized production amplitudes are independent of variations in the number of events among different bins, and therefore they are excellent starting values for neighboring bins[16].

\section{Calculation of Observables}

The acceptance-corrected number of events is after renormalization,

$$
\overline{N_{c}}\left({ }^{\varepsilon} V_{k \alpha}\right)=N_{o} \frac{N_{r}}{N_{\eta}} \sum_{k, \varepsilon, \alpha, \alpha^{\prime}}{ }^{\varepsilon} V_{k \alpha}{ }^{\varepsilon} V_{k \alpha^{\prime}}^{*} \Phi_{\alpha \alpha^{\prime}}
$$

The acceptance corrected number of events attributed to a state $|\alpha, \varepsilon\rangle$ is

$$
\overline{N_{|\alpha, \varepsilon\rangle}}=N_{o} \frac{N_{r}}{N_{\eta}} \sum_{k}\left|{ }^{\varepsilon} V_{k \alpha}\right|^{2 \varepsilon} \Phi_{\alpha \alpha},
$$

and the phase difference between two states $|\alpha, \varepsilon\rangle$ and $\left|\alpha^{\prime}, \varepsilon\right\rangle$ is

$$
\phi_{\alpha \alpha^{\prime} \varepsilon}=\arg \left(\sum_{k}^{\varepsilon} V_{k \alpha}{ }^{\varepsilon} V_{k \alpha^{\prime}}^{*}\right) .
$$

The above phase is only meaningful if states $|\alpha, \varepsilon, k\rangle$ and $\left|\alpha, \varepsilon, k^{\prime}\right\rangle$ are produced coherently. A measure of the coherence is given by

$$
{ }^{\varepsilon} C_{\alpha \alpha^{\prime}}=\frac{\left|\sum_{k}^{\varepsilon} V_{k \alpha}^{\varepsilon} V_{k \alpha^{\prime}}^{*}\right|}{\sqrt{\left(\left.\left.\sum_{k}\right|^{\varepsilon} V_{k \alpha}\right|^{2}\right)\left(\left.\left.\sum_{k}\right|^{\varepsilon} V_{k \alpha^{\prime}}\right|^{2}\right)}} .
$$

\section{Exotic Meson Candidates}

Studies of meson spectra via strong decays of hadrons provide insight regarding QCD at the confinement scale. These studies have led to phenomenological models for QCD such as the constituent quark model. However, QCD demands a much richer spectrum of meson states which includes extra states such as hybrids $(q \bar{q} g)$, multiquarks $(q \bar{q} q \bar{q})$, and glueballs ( $g g$ or $g g g$ ). Experiment E852 at Brookhaven National Laboratory is an experiment in meson spectroscopy configured to detect both neutral and charged final states of $\pi^{-} p$ collisions in a search for meson states incompatible with the constituent quark model.

The apparatus is located at the Multi-Particle Spectrometer (MPS) of Brookhaven's Alternating Gradient Synchrotron (AGS). For E852, the AGS delivered an $18 \mathrm{GeV} / c \pi^{-}$beam to a fixed liquid hydrogen target at the MPS. The MPS facility was augmented with additional detectors designed specifically for E852 and consists of 3 integral regions: target, charged tracking, and downstream regions(see Figure 3).

The target region is located in the middle of the MPS magnet and contains the following elements: a $30.5 \mathrm{~cm}$ long liquid hydrogen target, a four-layer cylindrical drift-proportional wire chamber, and a 198 block CsI(Ti) barrel veto detector. 


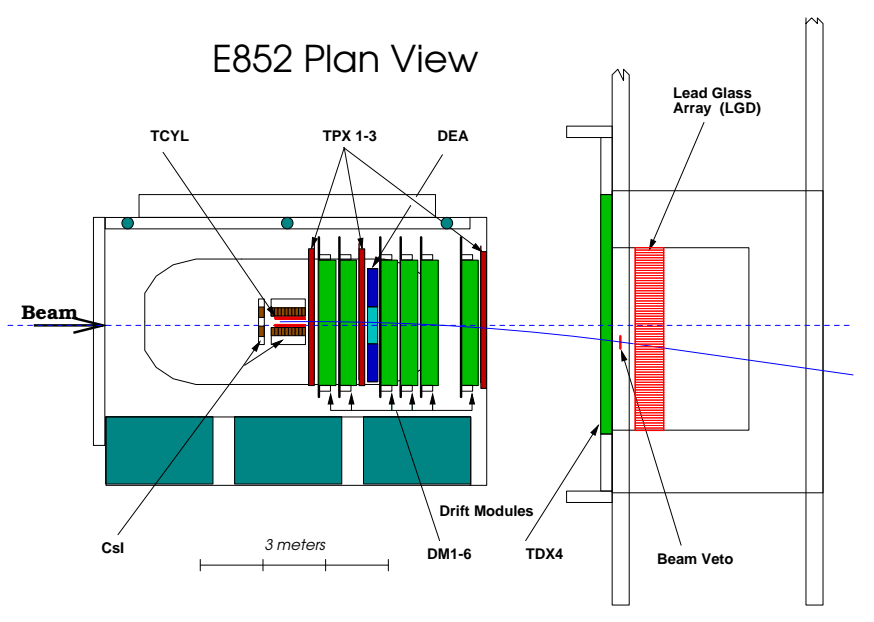

Figure 3: The plan view of E852 Apparatus at the MPS facility.

The downstream half of the MPS magnet houses the main components of the charged tracking region. It consists of 3 proportional wire chambers (TPX1-3) and 6 drift chambers modules(DM16) each with seven-layers. In addition to the tracking chambers, there are two scintillation veto counters (CPVB and CPVC) and a window-frame lead-scintillator sandwich veto detector (DEA).

The downstream region contains a 3000 element lead-glass calorimeter (LGD) for detecting and measuring the energy of the forward going gammas and a large drift chamber (TDX4) located directly in front of the LGD for tagging charged particles entering the LGD. A more detailed description of the E852 apparatus is given in Reference [17].

\subsection{The $\pi_{1}(1400)$ Exotic Meson}

The $\eta \pi$ system is particularly interesting in searching for exotic (or non- $q \bar{q}$ ) mesons because the system has spin $(\mathrm{J})$, parity $(\mathrm{P})$, and charge-conjugation $(\mathrm{C})$ in sequence $J^{P C}=0^{++}, 1^{-+}, 2^{++}, 3^{-+} \ldots$ for $l=0,1,2,3, \ldots$. (Here $l$ is the orbital angular momentum of the $\eta \pi$ system.) Hence a resonance with an $\eta \pi$ decay mode with odd $l$ is manifestly exotic.

In previous publications[3, 18], Brookhaven E852 presented evidence for an exotic meson produced in the reaction $\pi^{-} p \rightarrow \eta \pi^{-} p$ at $18 \mathrm{GeV} / c$ from an analysis of the $1994 \mathrm{E} 852$ data set. A large asymmetry in the angular distribution was observed indicating interference between $l$-even and $l$-odd partial waves. The $a_{2}(1320)$ was observed in the $J^{P C}=2^{++}$wave, as was a broad enhancement between 1200 and $1600 \mathrm{MeV} / c^{2}$ in the $1^{-+}$exotic wave. The observed phase difference between these waves shows that there was phase motion in addition to that due to $a_{2}(1320)$ decay. An analysis of the mass dependence of the partial waves shows that the data is well described by the interference between the $a_{2}(1320)$ and an exotic $1^{-+}$resonance with Mass $=\left(1370 \pm 16_{-30}^{+50}\right)$ $\mathrm{MeV} / \mathrm{c}^{2}$ and $\Gamma=\left(385 \pm 40_{-105}^{+65}\right) \mathrm{MeV} / \mathrm{c}^{2}$.

\subsection{The $\pi_{1}(1600)$ Exotic Meson}

E852 has performed a partial wave analysis of the reaction $\pi^{-} p \rightarrow \pi^{+} \pi^{-} \pi^{-} p[19]$. In summary, all expected well-known states $\left(a_{1}, a_{2}\right.$, and $\left.\pi_{2}\right)$ are observed. In addition, the natural parity exchange wave with manifestly exotic quantum numbers $J^{P C}=1^{-+}$shows structure in the inten- 
sity and phase motion which is consistent with a resonance at $1600 \mathrm{MeV} / \mathrm{c}^{2}$ decaying into the $\rho \pi$ channel. A mass dependent fit results in a resonance mass of $1593 \pm 8_{-47}^{+29} \mathrm{MeV} / c^{2}$ and a width of $168 \pm 20_{-12}^{+150} \mathrm{MeV} / \mathrm{c}^{2}$.

A subsuquent analysis studied the $\eta^{\prime} \pi^{-}$system produced in the reaction $\pi^{-} p \rightarrow \eta \pi^{+} \pi^{-} \pi^{-} p$. Figure 4a shows a clear signal for $\eta^{\prime} \rightarrow \eta \pi^{+} \pi^{-}$in the $\eta \pi^{+} \pi^{-}$mass spectrum, and shown in Figure $4 \mathrm{~b}$ is the accepted $\eta^{\prime} \pi^{-}$mass spectrum which exhibits a peak in the region of the $a_{2}(1320)$ and a broad peak near $1600 \mathrm{MeV} / \mathrm{c}^{2}$. The results of a partial wave analysis find two important partial waves: the $2^{++}$wave consistent with an $a_{2}(1320)$ signal and a broad higher mass structure; and a dominant $J^{P C}=1^{-+}$exotic wave which peaks at $1600 \mathrm{MeV} / c^{2}$.
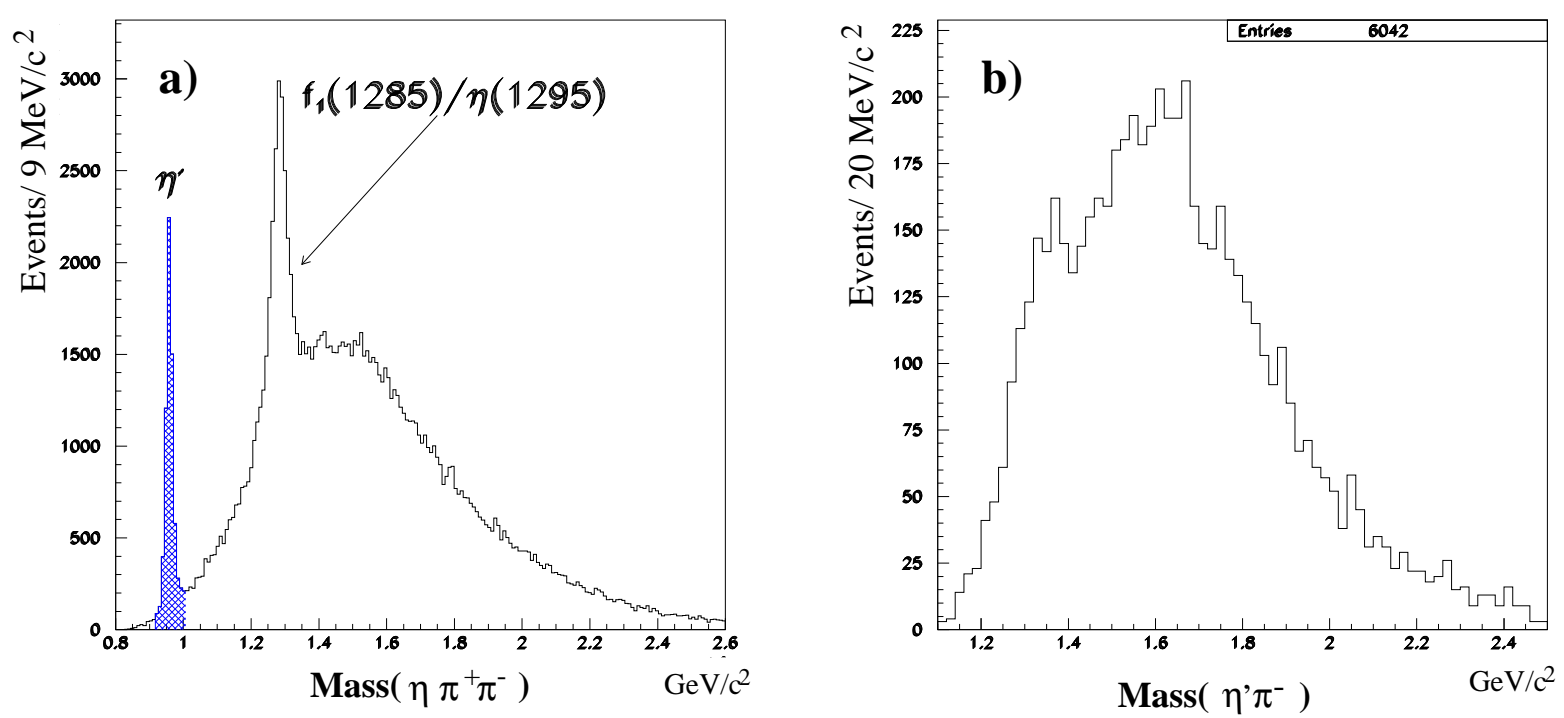

Figure 4: a) The accepted $\eta \pi^{+} \pi^{-}$effective mass distribution. b) The accepted $\eta^{\prime} \pi^{-}$effective mass distribution.

A study of the mass dependence of the PWA results finds three Breit-Wigner(BW) poles in the $2^{++}$wave and two BW poles in the $1^{-+}$wave. Figure 5 displays the PWA results(data points with errors) with the curves showing the results of the mass dependent fit.

Since the $1^{-+}$wave is dominated by a pole at $1600 \mathrm{MeV} / \mathrm{c}^{2}$ with a small contribution from a pole at $1400 \mathrm{MeV} / c^{2}$, an alternative fit, one with a poorer $\chi^{2} / D o F$ of 1.22 , finds that the $1^{-+}$wave can be described by the pole at $1600 \mathrm{MeV} / \mathrm{c}^{2}$ only. The results for the $2^{++}$poles and the $1^{-+}$pole at $1600 \mathrm{MeV} / \mathrm{c}^{2}$ are stable and are not affected by the parameterization of the $1^{-+}$low mass region.

In the Fux-tube model the lightest 1-+ isovector hybrid is predicted to decay primarily to $b_{1} \pi[11,12,13]$. The $f_{1} \pi$ branch is also expected to be large and many other decay modes are suppressed. This suppression is consistent with recent calculations showing $1 / \mathrm{Nc}$ behavior for decays to spin-zero mesons in the large-Nc limit of QCD[26].

Few experiments have addressed the $b_{1} \pi$ and $f_{1} \pi$ meson decay channels. The VES collaboration reported a broad $J^{P C}=1^{-+}$peak in $b_{1} \pi$ decay[29], and Lee, et al. [28] observed significant $J^{P C}=1^{-+}$signal in a $f_{1} \pi$ decay. In neither case was a definitive resonance interpretation of the 1-+ waves possible. Preliminary results from a later VES analysis show excitation of $\pi_{1}(1600)$ [27]. 

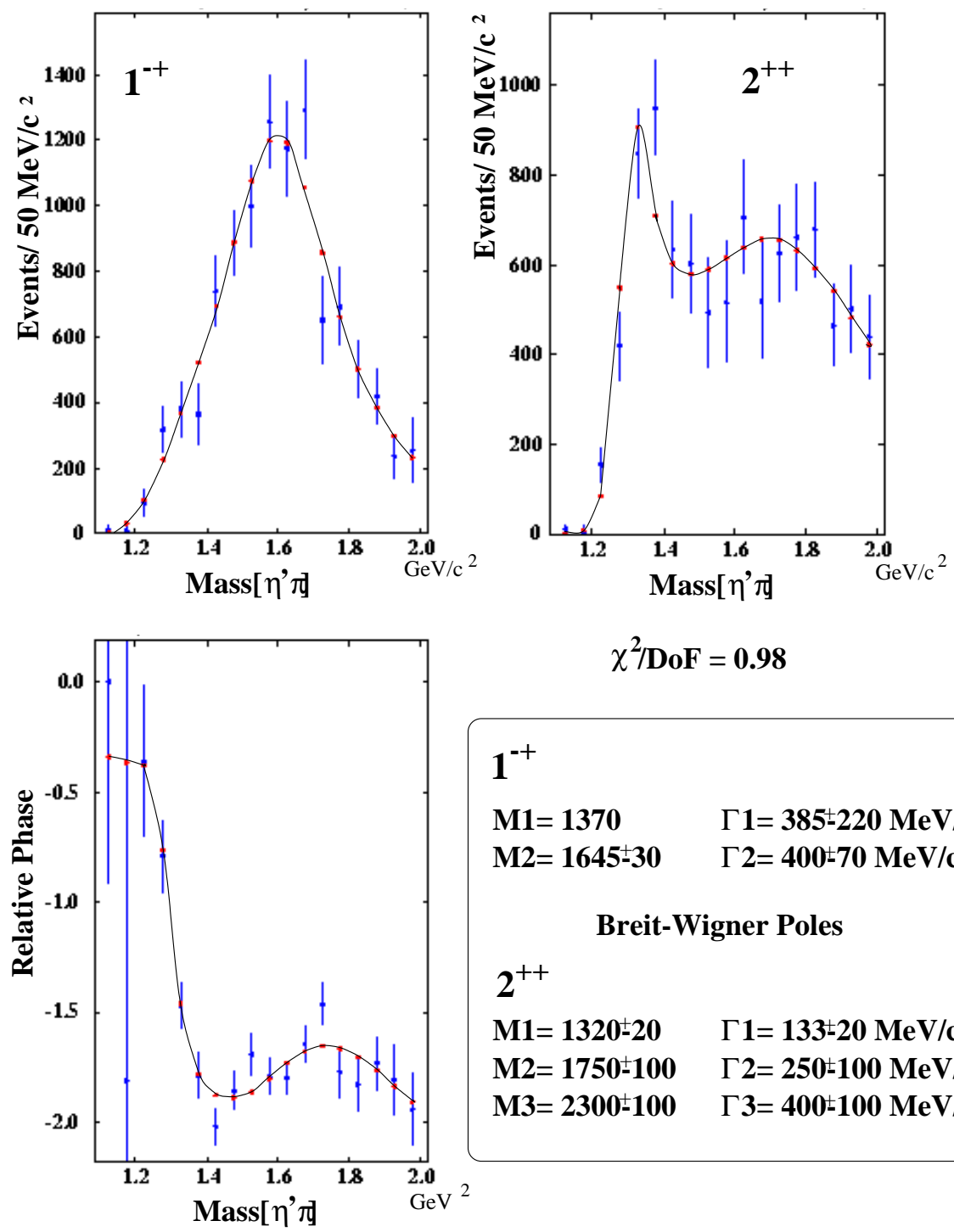

$\chi^{2} / \mathrm{DoF}=0.98$

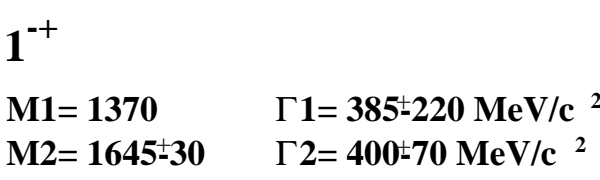

Breit-Wigner Poles

$2^{++}$

$M 1=1320 \pm 20 \quad \Gamma 1=133 \pm 20 \mathrm{MeV} / \mathrm{c}^{2}$

$\mathrm{M} 2=1750 \pm 100 \quad \Gamma 2=250 \pm 100 \mathrm{MeV} / \mathrm{c}^{2}$

$\mathrm{M3}=2300 \pm 100 \quad \Gamma 3=400 \pm 100 \mathrm{MeV} / \mathrm{c}^{2}$

Figure 5: The mass dependency of the partial wave intensities and relative phases. Results of the PWA are given as data points with errors whereas results of a mass dependent fit assuming 2 poles in the $1^{-+}$and 3 poles in the $2^{++}$are shown as curves.

Significant $b_{1} \pi$ strength for $\pi_{1}(1600)$ was also reported[30]. Recently BNL experiment E852 reported a measurement of $f_{1} \pi$ and $b_{1} \pi$ decays for $\pi_{1}(1600)$ and $\pi_{1}(2000)$ [31, 32].

\section{GlueX: The Science of Quark Confinement \& Gluonic Excitations}

A recent effort at Jefferson Lab, in conjunction with the plans for the energy upgrade of CEBAF(see Figure 6), is the development of a state-of-the-art hermetic spectrometer-the GlueX project(formerly know as the Hall D project). One of the scientific motivations for upgrading CE- 


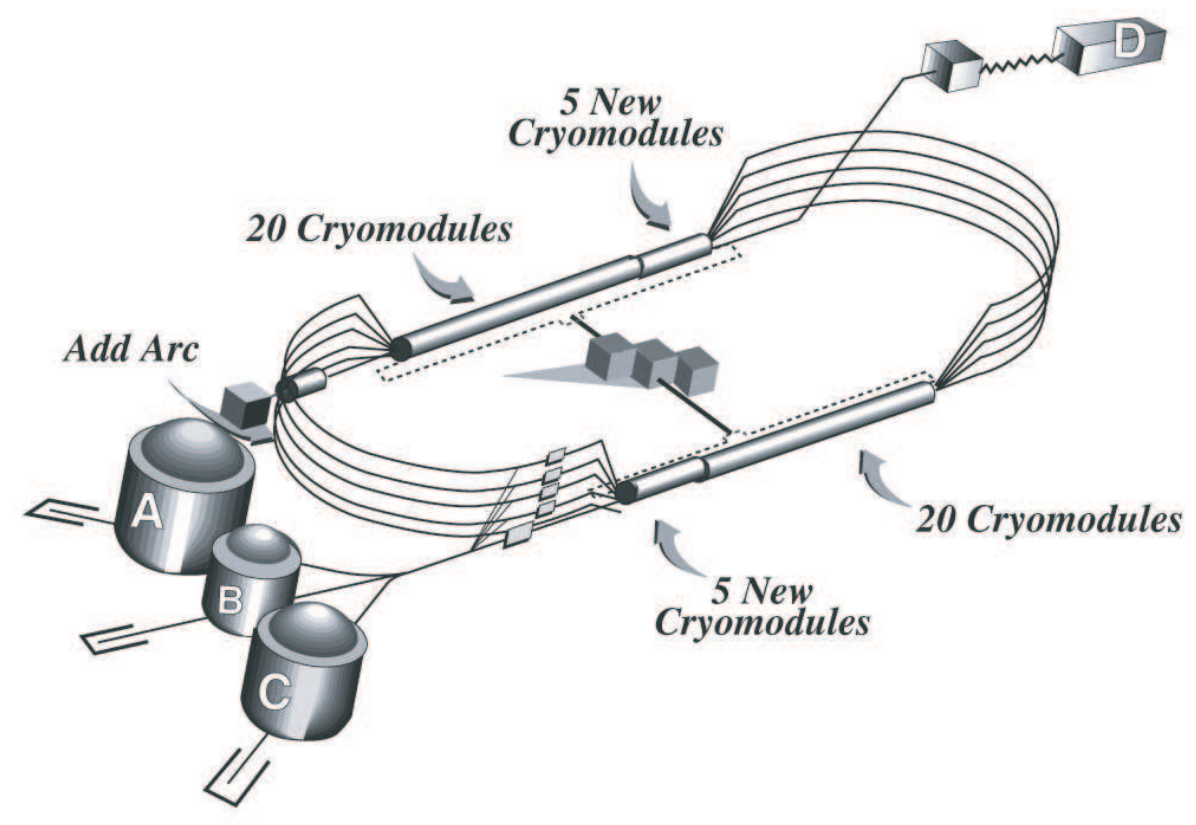

Figure 6: The CEBAF multi-pass electron accelerator at Jefferson Lab. Shown are three existing experimental Halls (A, B, \& C) and the planned Hall D.

BAF is a high-statistics definitive study of the photoproduction of mesons with masses below the $c \bar{c}$ threshold in a search for new forms of hadronic and gluonic matter. After many years of searching for gluonic excitation produced via hadronic probes, only now are we finding some promising candidates. An unexplored search area is the photoproduction of light quark meson states. Since the photon can be thought of as a virtual vector meson with quark spins aligned, it is a probe distinct from the traditional hadronic probes of pions, kaons, and protons. Within the Flux-tube model, the production of gluonic excitations via photon interaction is expected to produce a wealth of states with manifestly exotic $J^{P C}$ 's ( $q \bar{q}$ forbidden quantum numbers). The goal of the GlueX experiment is a mapping of the spectrum of gluonic excitations with the ultimate objective being a quantitative understanding of the nature of confinement in QCD. To achieve this goal a hermetic detector, the GlueX spectrometer(see Figure 7), optimized for amplitude analysis, will be constructed in a new experimental hall (Hall D). A tagger facility will produce $9 \mathrm{GeV}$ linearly polarized photons via coherent bremsstrahlung radiation of $12 \mathrm{GeV}$ electrons through a diamond wafer. To achieve $12 \mathrm{GeV}$ electrons CEBAF will be upgraded to $12 \mathrm{GeV}$ with additional cryomodules, modified arcs and an additional arc. Critical Decision 0 (CD-0) for the upgrade and GlueX was awarded by the Department of Energy (DOE) in 2004 followed by Critical Decision 1 (CD-1) approval in 2006. The GlueX collaboration was formed in 1998. The project has been reviewed externally and by the Jefferson Lab PAC. The GlueX management has been in place since 2000 with a Spokesman, Deputy-spokesman, Hall D group leader and an elected Collaboration Board.

\section{HyCLAS: The Search for Gluonic Hybrid Mesons with the JLAB CLAS Facility}

One of Jefferson Lab's most important missions is the study of QCD at intermedate energies. 


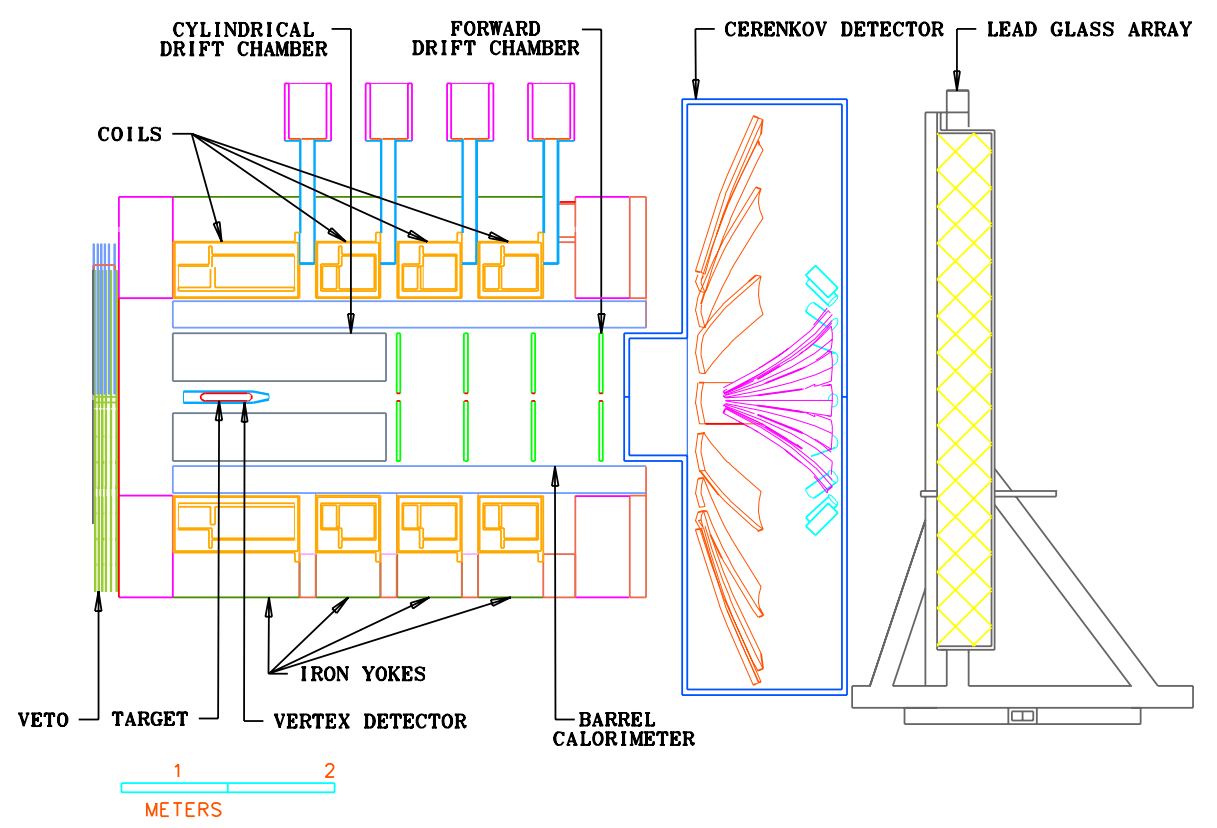

Figure 7: An overview of the GlueX detector. The major subsystems are labled. The photon beam enters the apparatus as draw from the left side of the figure.

Meson spectroscopy is one of the leading ways to study QCD at the confinement scale. Exotics, Hybrids, and Strangeonia are poorly known, and they represent the next frontier in hadronic physics. The spectroscopy of mesons in the 1 to $2 \mathrm{GeV} / \mathrm{c}^{2}$ mass range will provide insight into these new forms of hadronic matter, and thus aid in the study of QCD at the realm of confinement. Tantalizing evidence for "gluonic hybrid" states exists, but the interpretation is controversial. Future projects such as GlueX at Jefferson Lab and PANDA at GSI will eventually provide definitive measurements of exotic states, but it will be several years before these experiments will acquire data. Jefferson Lab offers an unique opportunity to undertake the study of meson spectroscopy at intermediate energies. And current studies at CLAS are showing the feasibility of using CLAS as a meson spectrometer for few-body final states.

Motivated by these and recent experimental results for gluonic hybrid candidates and from recent theoretical Lattice QCD and Flux-tube model calculations, Paul Eugenio (as spokesperson/contact person for JLAB E04-005) led the effort to develop a program for a high statistical search for gluonic hybrid mesons and strangeonia ${ }^{1}$ utilizing a high energy photon beam at CLAS. Given the current limited acceptance of CLAS, E04-005 will concentrate on important final states but with the focus on channels with a limited number of three to four charged particles in the final state.

The Jefferson Laboratory Program Advisory Committee (JLAB PAC 25) held its 25th meeting on January 12-18, 2004. The PAC, in response to the charge from the JLab Director, Dr. Christoph Leemann, reviewed and made recommendations for proposals and letters of intent submitted by Jefferson Lab users. Our proposal (E04-005) was awarded 35 days of beam-time received an "A"

\footnotetext{
${ }^{1}$ mesonic states with hidden strange quark content
} 
approval rating. In its report, the PAC stated,

In a measurement in Hall B, the search for new forms of hadronic matter in the meson sector will complement the ongoing investigations in the baryon sector. The chances of identifying exotic meson states with this experiment look very promising. The progress made by the proposers in establishing phase shift analysis procedures in the analysis of CLAS data has been remarkable. This is an experiment of exceptional promise, putting Jefferson Laboratory at the forefront of exotic meson spectroscopy.

The program will perform a high statistical partial wave analysis of meson spectroscopy data acquired using CLAS. The program will study meson systems in the mass ranging from 1.0 to 2.3 $\mathrm{GeV}$ in reactions having three to four charged particles in the final state of the form:

$$
\begin{gathered}
\gamma p \rightarrow p \pi^{+} \pi^{-} \pi^{0} \\
\gamma p \rightarrow n \pi^{+} \pi^{+} \pi^{-} \\
\gamma p \rightarrow p \phi \eta \rightarrow p K^{+} K^{-} \eta \\
\gamma p \rightarrow n \phi \pi^{+} \rightarrow n K^{+} K^{-} \pi^{+} \\
\gamma p \rightarrow n \pi^{+} \pi^{-} \pi^{+} \pi^{-} \\
\gamma p \rightarrow \Delta^{++} \eta \pi^{-} \rightarrow p \pi^{+} \pi^{-} \eta \\
\gamma p \rightarrow \Delta^{++} \omega \pi^{-} \rightarrow p \pi^{+} \omega \pi^{-} \\
\gamma p \rightarrow n \eta / \pi^{+} \rightarrow n \pi^{+} \pi^{-} \eta \pi^{+} \\
\gamma p \rightarrow p K^{ \pm} K^{0} \pi^{\mp} \\
\gamma p \rightarrow p p \bar{p}
\end{gathered}
$$

The photon energy required to reach the production threshold for a meson $\mathrm{X}$ of mass $m_{X}$ is ( $M_{t}$ is the proton target mass and $M_{r}$ is the baryon recoil mass)

$$
E_{\gamma}=\frac{m_{X}^{2}+M_{r}^{2}-M_{t}^{2}+2 m_{X} M_{r}}{2 M_{t}}
$$

Therefore, with a 5-6 $\mathrm{GeV}$ photon beam we can reach up to 2.3-2.5 $\mathrm{GeV}$ in meson masses. However, since we need to populate enough phase space for the decay products of the mesons to be experimentally measurable, at least an extra $0.5 \mathrm{GeV}$ from the threshold energy is required. For example, for the $\phi(1850)$ decays to be detected, it will be necessary for photon energies of around $5 \mathrm{GeV}$. Higher beam energies are desirable to isolate the baryon and meson decays in phase space. However, in the case of strangeness production, the number of strange baryons contributing to the background are less and better isolated than for $n \bar{n}$ backgrounds. In some cases, i.e., $K \bar{K} \pi$ decay channel, baryon backgrounds are not present. 


\section{References}

[1] N. Isgur, R. Kokoski, and J. Paton, Phys. Rev. Lett. 54, 869 (1985).

[2] P. Lacock, et.al.(UKQCD Collaboration), Phys. Lett. B401, 308 (1997).

[3] D. R. Thompson et. al. (BNL-E852 Collaboration,) Phys. Rev. Lett. 79, 1630 (1997).

[4] G. S. Adams et. al. (BNL-E852 Collaboration), Phys. Rev. Lett. 81, 5760 (1998).

[5] See contributions from both A. V. Popov and D. V. Amelin, 9th International Conference on Hadron Spectroscopy: HADRON01 Protvino, Russia 2001.

[6] F. Close and P. Page, Phys. Rev. D52, 1706 (1995).

[7] A. Szczepaniak and M. Swat, Phys. Lett. B516, 72 (2001).

[8] N. Isgur and J. Paton, Phys. Rev. D31, 2910 (1985).

[9] GlueX/Hall D Collaboration The GlueX/Hall D Design Report 38 (2002).

[10] T. Barnes, F. E. Close, and E.S. Swanson, Phys.Rev. D52 5242 (1995).

[11] G. R. Blackett, et al, arXiv.org e-Print Archive hep-ex/9708032 (1997).

[12] S. U. Chung, Brookhaven Report BNL-QGS-97-041 (1995).

[13] P. Eugenio, Int. J. Mod. Phys. A 18, 487 (2003).

[14] James, F., CERN Program Library Long Writeup D506 Ver.94.1 (1994).

[15] Orear, Jay, Notes on Statistics For Physicists, Revised CLNS 82/511 (1982).

[16] Chung, S. U., Brookhaven Report BNL-QGS-93-05 (1995).

[17] S. Teige et al., Phys. Rev., D59, 12001, (1999)

[18] S.U. Chung et al., Phys. Rev., D, Accepted, (1999)

[19] G. S. Adams et. al., Phys. Rev. Lett. 81, 5760, (1998)

[20] Corden et al., Nucl. Phys. B144, 153, (1978)

[21] Particle Data Group, Phys. Rev. D54 (1996) 1.

[22] D. Amelin et al., Phys. Lett. B356, 595, (1995).

[23] N. Isgur and J. Paton, Phys. Rev. D31, 2910 (1985).

[24] F.E. Close and P.R. Page, Nucl. Phys. B443, 233 (1995).

[25] Philip R. Page, Eric S. Swanson, and Adam P. Szczepaniak, Phys. Rev. D59, 034016-1 (1999).

[26] Philip R. Page, Phys. Rev. D70, 016004(2004).

[27] D.V. Amelin, et al., Yad. Fiz. 62, 487 (1999); ibid, Phys.Atom.Nucl.62, 445 (1999).

[28] J.H. Lee, et al., Phys. Lett. B323, 227 (1994).

[29] Valery Dorofeev, et al., AIP Conf. Proc. 619, 143 (2002).

[30] C.A. Baker, et al., Phys. Lett. B563, 140 (2003).

[31] M. Lu et al., Phys. Rev. Lett. 94, 032002, (2005).

[32] J. Kuhn,et al., Phys. Lett.B595, 109 (2004). 